\title{
The Role of Silver Additions in Formation of Sn-Bi-Ag Semiconductor Alloys by Rapid Solidification
}

\author{
Tarek El Ashram ${ }^{1,2}$ \\ ${ }^{1}$ Physics Department, Faculty of Science, Port Said University, Port Said, Egypt \\ ${ }^{2}$ Physics Department, Preparatory Year Deanship, Jazan University, Jazan, Kingdom of Saudi Arabia \\ Email: tnelashram@gmail.com
}

Received 13 February 2015; accepted 4 March 2015; published 10 March 2015

Copyright (@ 2015 by author and Scientific Research Publishing Inc.

This work is licensed under the Creative Commons Attribution International License (CC BY). http://creativecommons.org/licenses/by/4.0/

(c) (i) Open Access

\begin{abstract}
Five alloys Sn-5Bi-xAg $(x=0,1,2,3$, and 4 in at $\%)$ are produced by rapid solidification using melt-spinning technique. From temperature dependence of electrical resistivity (TDR), it is found that the Sn-5Bi-xAg $(x=1,2,3,4$ in at \%) rapidly solidified by melt spinning technique are narrow band semiconductor alloys. The energy gap $E_{g}$ decreases by increasing Ag concentration from 203 $\mathrm{meV}$ for $\mathrm{Sn-5Bi-1Ag}$ to $\mathbf{9 7 . 5} \mathrm{meV}$ for $\mathrm{Sn-5Bi-4Ag}$ alloy. From $\mathrm{x}$-ray diffraction analysis (XRD), it is found that the Hume-Rothery condition for phase stability is not satisfied for this alloy.
\end{abstract}

\section{Keywords}

Rapid Solidification, Semiconductor, Energy gap, Resistivity, Sn-Bi-Ag Alloys

\section{Introduction}

The production of semiconductor alloys by rapid solidification is simple, uncomplicated and economic. By this method, we can directly obtain thin semiconductor wafers available for manufacturing semiconductor devices. Also, the semiconductors prepared by rapid solidification exhibit considerable improvements over alloys made from conventional materials. The thermoelectric materials fabricated by rapid solidification displayed a high figure of merit [1]. Rapid solidification prevents rejection of extra solute atoms and thus prevents precipitation from a solid solution, which in turn increases the solid solubility. The production of semiconductor alloys by rapid solidification depends mainly on the type of alloy system used. Most of these alloys are based on group V, such as $\mathrm{Bi}$. The solute atoms play an important role in formation of semiconductors by rapid solidification. In 
this respect, a series of rapidly solidified Bi based semiconductor alloys were produced using melt spinning technique [2]-[4]. They found that the semiconducting behavior depended on the type of the solute atoms added to $\mathrm{Bi}$. The alloys contain solute atoms with odd valencies such as $\mathrm{Ag}(+1)$, $\mathrm{Al}(+3)$ and $\mathrm{Sb}(+5)$ have semiconducting behavior by contrast all alloys contain solute atoms with even valencies such as $\mathrm{Zn}(+2)$ and Sn (+4) have metallic behavior. The produced semiconductor alloys are narrow band semiconductors. The band gap was decreased by increasing valency from $225.1 \mathrm{meV}$ for Bi-Ag system to $12.7 \mathrm{meV}$ for Bi-Sb system. Also some alloys based on $\mathrm{Bi}$, such as $\mathrm{Bi}$-Sb were found to be semiconductors even though they were produced by conventional methods [5]-[7]. It was found by [8] that by alloying Bi with Sn, a large increase in resistivity was obtained and the temperature coefficient of resistivity was found to be zero. The present work is an attempt to study the effect of rapid solidification on the electrical properties of alloy system based on an element in group IV such as tin ( $\beta$-Sn). Group IV contains the elemental semiconductors $\mathrm{Si}, \mathrm{Ge}$, and $\alpha$-Sn, (the allotrope of Sn stable below $13.2^{\circ} \mathrm{C}$ ). The three previous semiconductor elements $\mathrm{Si}, \mathrm{Ge}$ and $\alpha$-Sn have the same crystal structure which is diamond cubic. $\beta$-Sn (the stable allotrope of $\mathrm{Sn}$ above $13.2^{\circ} \mathrm{C}$ ) is metallic and its crystal structure is body centered tetragonal. Pure tin $(\beta$-Sn) was rapidly solidified using melt spinning technique and no amorphous or non-equilibrium crystalline phases was formed [9]. Also it was found that its resistivity at room temperature was increased and its temperature coefficient of resistivity (TCR) was decreased in comparison with conventional pure $\beta$-Sn. Therefore, the aim of the present work is to study the effect of rapid solidification using melt spinning technique on the structure and electrical properties of Sn-Bi. Also the effect of silver (Ag) additions on the structure and electrical properties of Sn-Bi alloy will be studied.

\section{Experimental Procedures}

The materials used in the present work were $\mathrm{Sn}, \mathrm{Bi}$, fragments and $\mathrm{Ag}$ wires, the starting purity were better than 99.99\%. Five alloys Sn-5Bi-xAg ( $x=0,1,2,3$, and 4 in at \%) were produced by a single copper roller (200 mm in diameter) melt spinning technique. The process parameters such as the ejection temperature, and the linear speed of the wheel were fixed at $873 \mathrm{~K}$ and $30.4 \mathrm{~ms}^{-1}$ respectively. X-ray diffraction analysis (XRD) was carried out with a XPERT-PRO X-ray diffractometer, using $\mathrm{Cu}-\mathrm{K}_{\alpha}$ radiation $(\lambda=1.5406 \AA)$. Differential Scanning Calorimetry (DSC) was carried out in a Shimadzu (DSC-60) with heating rate $10 \mathrm{~K} \cdot \mathrm{min}^{-1}$. The temperature dependence of resistivity (TDR) was measured by four probe method using microhmmeter of type BS407. The BS407 uses a four terminal measurement system via high quality Kelvin Clip leads with sensitivity is $1 \mu \Omega$. The heating range starts from room temperature up to $530 \mathrm{~K}$ with heating rate about $5 \mathrm{~K} \cdot \mathrm{min}^{-1}$.

\section{Results and Discussion}

\subsection{Structure}

Figure 1 shows the $x$-ray diffraction XRD patterns for as-quenched melt-spun Sn-5Bi-xAg, $(x=0,1,2,3$, and 4 in at \%) alloys. The XRD for Sn-5Bi alloy is shown in Figure 1(a), Bi is precipitated as indicated by (012) peak and all the other peaks are for $\mathrm{Sn}$. The matrix is $\beta$-Sn solid solution, the unit cell of $\beta$-Sn is body centered tetragonal (I4 $/$ amd). The crystal structure of $\mathrm{Bi}$ is rhombohedral-hexagonal (S.G.: R3m) with $\boldsymbol{a}=4.5491, \boldsymbol{c}=$ $11.9485 \AA$.

Figure 1(b) shows for Sn-5Bi-1Ag alloy the same structure, i.e. precipitation of $\mathrm{Bi}$ in the $\mathrm{Sn}$ matrix as indicated by (012) peak. For Sn-5Bi-2Ag alloy (Figure 1(c)) the peak due to Bi is disappeared and instead a peak due to $\mathrm{Ag}_{3} \mathrm{Sn}$ (211) is appeared. As the Ag concentration increases the intensity of the peak due to $\mathrm{Ag}_{3} \mathrm{Sn}$ increases (Figure 1(d) and Figure 1(e)). The crystal structure of $\mathrm{Ag}_{3} \mathrm{Sn}$ is orthorhombic (S.G.: Pmmn) with lattice parameters $\boldsymbol{a}=5.9680, \boldsymbol{b}=4.7802$, and $\boldsymbol{c}=5.1843$. The detail of XRD analysis is shown in Table 1.

The axial ratio $\boldsymbol{c} / \boldsymbol{a}$ is very important parameter in determination the properties of Sn-based alloys since the work of [10]. Figure 2(a) shows the variation of the axial ratio $\boldsymbol{c} / \boldsymbol{a}$ with Ag concentration. The lattice parameters $\boldsymbol{a}, \boldsymbol{c}$, and the axial ratio $\boldsymbol{c} / \boldsymbol{a}$ for pure Sn rapidly solidified were found by [9] to be $5.809 \AA$, $3.169 \AA$, and 0.5455 respectively.

The addition of Bi to Sn expands the $\beta$-Sn tetragonal unit cell, both $\boldsymbol{a}$ and $\boldsymbol{c}$ are increased (see Table 1) and the $\boldsymbol{c} / \boldsymbol{a}$ ratio is decreased in agreement with [10]. It is evident that the addition of Ag increases the axial ratio from 0.5436 to 0.5464 in agreement with [10]. Since low valency atom like Ag increases the axial ratio of $\beta$-Sn tetragonal unit cell and high valency atom decreases it. Figure 2(b) shows the variation of the volume of the 


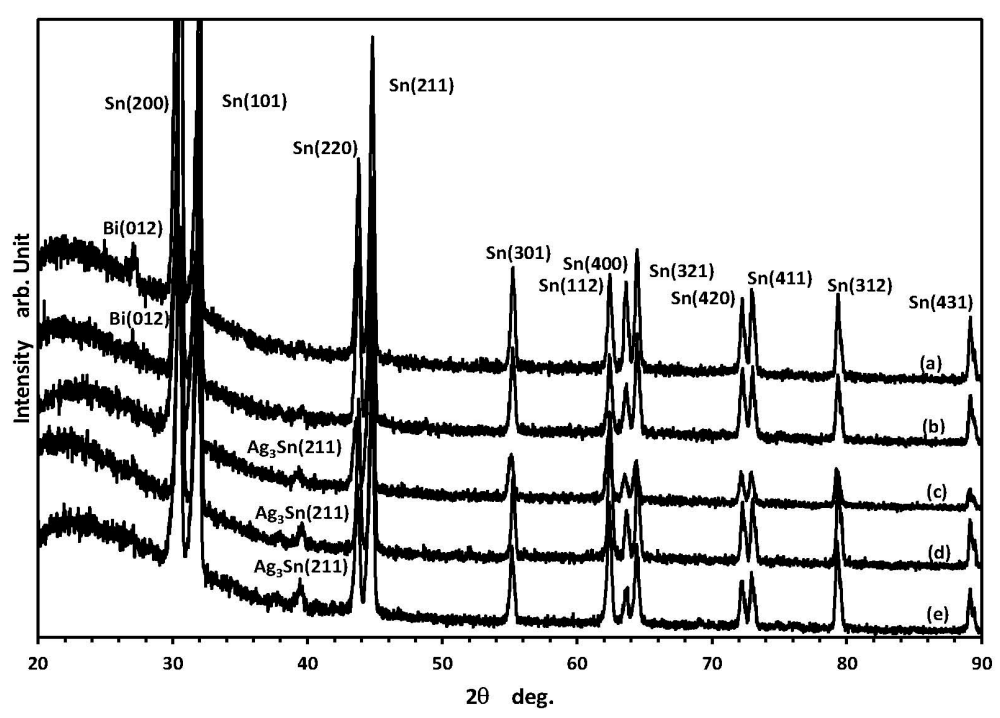

Figure 1. The XRD patterns for as-quenched melt-spun alloys. (a) Sn-5Bi; (b) Sn-5Bi-1Ag; (c) Sn-5Bi-2Ag; (d) Sn-5Bi-3Ag; and (e) Sn-5Bi-4Ag in at \%.

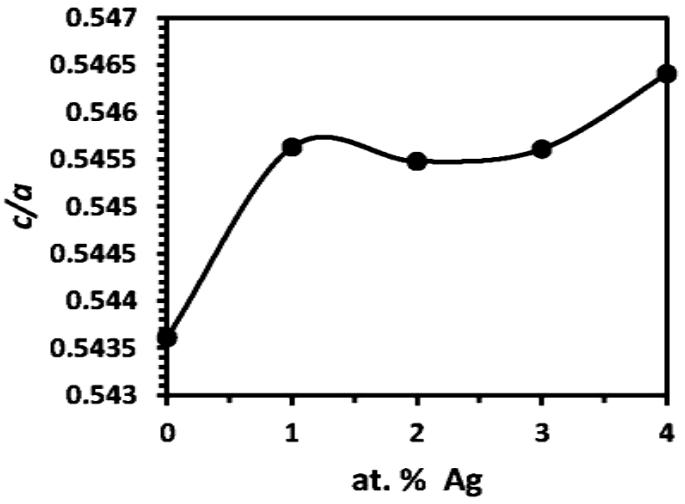

(a)

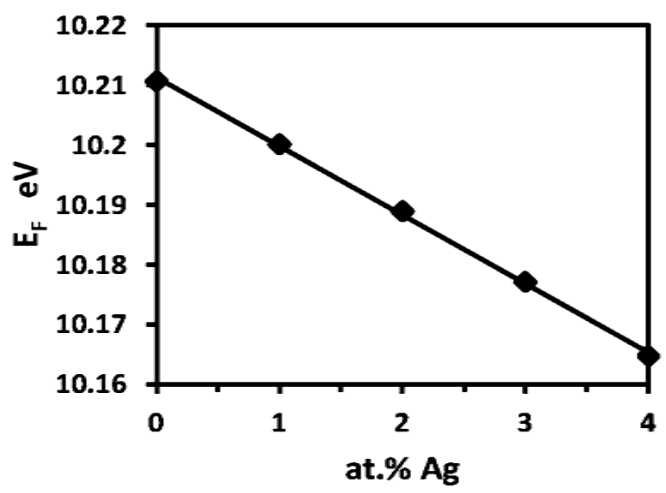

(c)

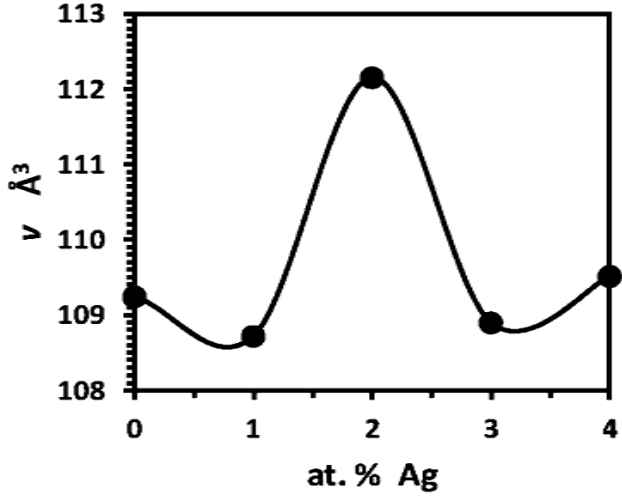

(b)

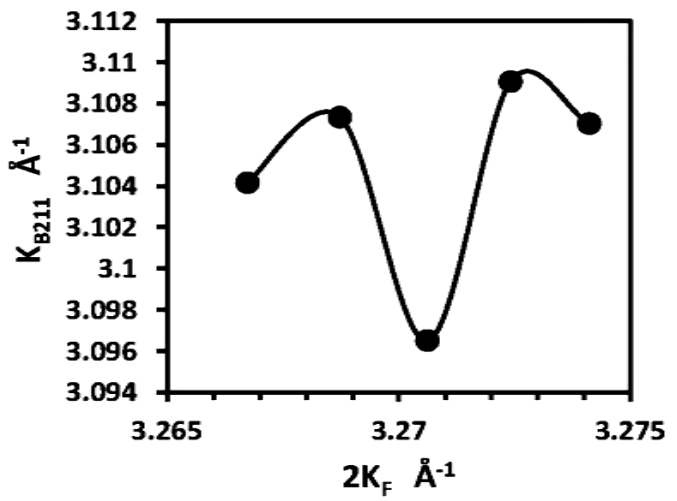

(d)

Figure 2. (a) The variation of $\boldsymbol{c} / \boldsymbol{a}$ with Ag concentration; (b) The variation of $\boldsymbol{v}$ with Ag concentration; (c) The variation of $\mathrm{E}_{\mathrm{F}}$ with Ag concentration; and (d) The variation of $\mathrm{K}_{\mathrm{B} 211}$ with $\mathrm{k}_{\mathrm{F}}$.

unit cell $v$ of the $\beta$-Sn matrix with Ag concentration. The volume of unit cell has a maximum value $112.15 \AA^{3}$ at 2 at \% Ag. Figure 2(c) shows the variation of Fermi energy $E_{F}$ with Ag concentration. It is evident that Fermienergy decreases by increasing $\mathrm{Ag}$ concentration from $10.21 \mathrm{eV}$ to $10.16 \mathrm{eV}$. This decrease is due to that $\mathrm{Ag}$ 
Table 1. The $\mathrm{x}$-ray diffraction details for as-quenched melt spun Sn-5Bi-xAg ( $\mathrm{x}=0,1,2,3$, and 4 in at \%) alloys.

\begin{tabular}{|c|c|c|c|c|c|c|c|}
\hline \multirow{2}{*}{ Alloy } & \multirow{2}{*}{$\begin{array}{l}\text { Phases present in } \\
\text { Sn }\end{array}$} & \multirow{2}{*}{ hkl } & \multicolumn{4}{|c|}{ The lattice parameters of the $\beta$-Sn matrix } & \multirow{2}{*}{ Crystal structure } \\
\hline & & & $a \AA$ & $c \AA$ & $c / a$ & $v \AA^{3}$ & \\
\hline Sn-5Bi & $\mathrm{Bi}$ & (012) & 5.8573 & 3.1841 & 0.5436 & 109.24 & Rho.-hex. (S.G.: $R \overline{3} m$ ) \\
\hline Sn-5Bi-1Ag & $\mathrm{Bi}$ & $(012)$ & 5.8407 & 3.1868 & 0.5456 & 108.71 & Rho.-hex. (S.G.: $R \overline{3} m$ ) \\
\hline Sn-5Bi-2Ag & $\mathrm{Ag}_{3} \mathrm{Sn}$ & (211) & 5.9021 & 3.2194 & 0.5454 & 112.15 & Ortho. (S.G.: Pmmn) \\
\hline Sn-5Bi-3Ag & $\mathrm{Ag}_{3} \mathrm{Sn}$ & (211) & 5.8440 & 3.1885 & 0.5456 & 108.89 & Ortho. (S.G.: Pmmn) \\
\hline Sn-5Bi-4Ag & $\mathrm{Ag}_{3} \mathrm{Sn}$ & (211) & 5.8522 & 3.1976 & 0.5464 & 109.51 & Ortho. (S.G.: Pmmn) \\
\hline
\end{tabular}

atom with valency +1 replaces $S n$ atom with valency +4 . Figure $2(d)$ shows the variation of the diameter of Brillouin zone $\mathrm{K}_{\mathrm{B} 211}$ in the (211) direction with the diameter of Fermi sphere $2 \mathrm{k}_{\mathrm{F}}$. It is evident that the Hume-Rothery condition for phase stability is not satisfied for this alloy.

\subsection{Thermal Analysis}

Figure 3 shows the DSC curves for as-quenched melt-spun pure Sn-5Bi-x Ag ( $\mathrm{x}=0,1,2,3$, and 4 in at \%) alloys. A transition occurs at about $413 \mathrm{~K}$ for all alloys; however the endothermic peak for this transition decreases by increasing Ag concentration. This transition may be due to the eutectic reaction which starts at $413 \mathrm{~K}$. Finally, the endothermic peak due to melting was obtained, from which the melting temperature $T_{m}$ and the enthalpy of fusion $\Delta H_{m}$ have been determined. $\Delta H_{m}$ was obtained from the integral under the DSC peak of melting as given by Equation (1);

$$
\Delta H_{m}=\int_{T_{0}}^{T_{f}} C_{p} \mathrm{~d} T
$$

where; $C_{p}$ is the heat capacity at constant pressure, $T_{0}$ and $T_{f}$ are known as onset melting temperature and final melting temperature of the specimen, respectively. Table 2 shows the detail of the DSC results. It is evident that the melting temperature decreases by increasing $\mathrm{Ag}$ concentration from $492.29 \mathrm{~K}$ for Sn-5Bi to $483.58 \mathrm{~K}$ for Sn-5Bi-4Ag. The enthalpy of fusion $\Delta H_{m}$ decreases by increasing $\mathrm{Ag}$ concentration from $57.27 \mathrm{~kJ} \cdot \mathrm{kg}^{-1}$ for Sn-5Bi to $47.5 \mathrm{kJkg}^{-1}$ for Sn-5Bi-3Ag.

\subsection{Electrical Properties}

Figure 4(a) shows the temperature dependence of resistivity obtained for as-quenched melt-spun Sn-5Bi alloy in the temperature range from 300 to $500 \mathrm{~K}$. The behavior is metallic i.e. the resistivity increases by increasing temperature. The resistivity at room temperature $\rho$ is found to be $4.56 \times 10^{-6} \Omega \mathrm{m}$.

The resistivity at room temperature for pure Sn rapidly solidified was found to be $0.166 \times 10^{-6} \Omega \mathrm{m}$ [5]. This means that the addition of Bi causes large increase in the resistivity. This increase in the resistivity is attributed to two reasons; the first is the presence of $\mathrm{Bi}$ atoms in Sn lattice which acts as a scattering center for the conduction electrons. The second is due to the precipitation of $\mathrm{Bi}$ as a distinct phase which has high resistance $(2.09 \mathrm{x}$ $10^{-6} \Omega \mathrm{m}$ ) according to [3]. The temperature coefficient of resistivity TCR was calculated to be $9.15 \times 10^{-3} \mathrm{~K}^{-1}$ (see Table 3). The TCR is increased due to the addition of Bi since TCR for pure Sn rapidly solidified was found to be $3.23 \times 10^{-3} \mathrm{~K}^{-1}$ [9].

Figure 4(b) shows the temperature dependence of resistivity (TDR) obtained for as-quenched melt-spun Sn-5Bi-xAg ( $x=1,2,3$, and 4 in at \%) alloys. It is evident that the behavior is semiconducting for all alloys containing Ag i.e. the resistivity decreases by increasing temperature. The variation of resistivity at room temperature $\rho$ with Ag concentration is shown in Figure 4(c). The resistivity at room temperature $\rho$ decreases by increasing Ag concentration. This decrease in resistivity can be attributed to the increase in the axial ratio c/a due the addition of $\mathrm{Ag}$ as shown in Figure 2(a). The energy gap $\mathrm{E}_{\mathrm{g}}$ was calculated for the semiconducting alloys and the result is shown in Figure 4(d). The energy gap $E_{g}$ decreases by increasing Ag concentration from 203 $\mathrm{meV}$ for $\mathrm{Sn}-5 \mathrm{Bi}-1 \mathrm{Ag}$ to $97.5 \mathrm{meV}$ for $\mathrm{Sn}-5 \mathrm{Bi}-4 \mathrm{Ag}$ (see Table 3). The semiconducting behavior may be explained as the following; both $\mathrm{Bi}$ and $\mathrm{Ag}$ atoms are dissolved in Sn lattice, the Ag atoms provide a donor level near the conduction band. Electrons from this level are raised to the conduction band by thermal agitation. Therefore the total number of carriers increases with temperature which in turn decreases the resistivity. 


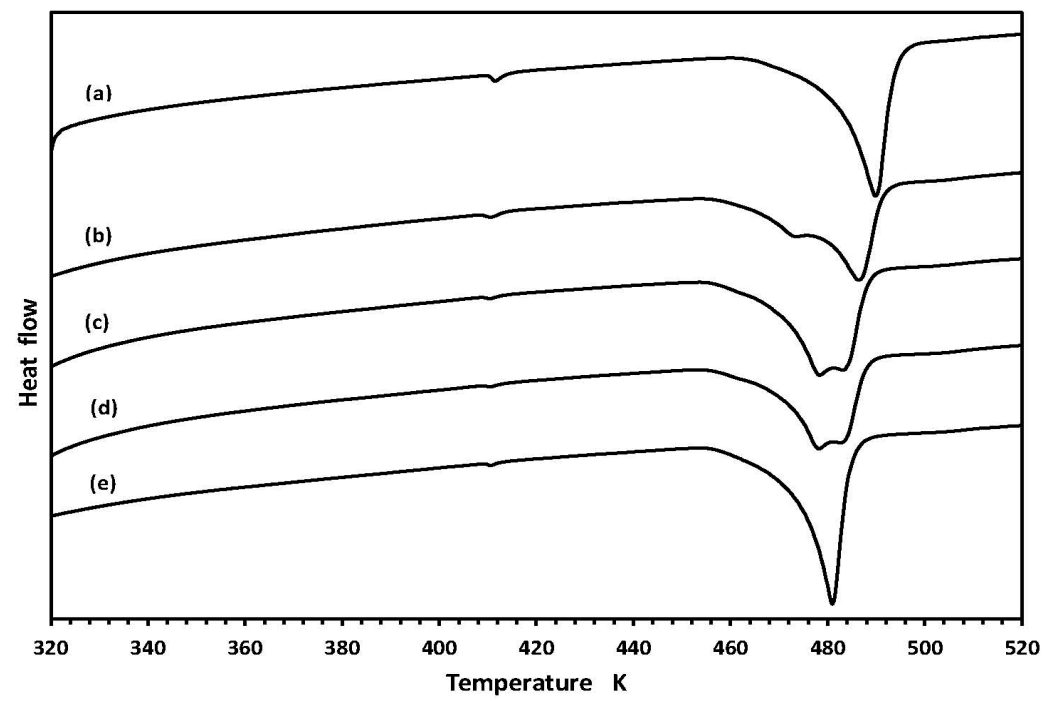

Figure 3. The DSC curves for as-quenched melt-spun alloys. (a) Sn-5Bi; (b) Sn-5Bi-1Ag; (c) Sn-5Bi-2Ag; (d) $\mathrm{Sn}-5 \mathrm{Bi}-3 \mathrm{Ag}$, and (e) Sn-5Bi-4Ag in at \%.

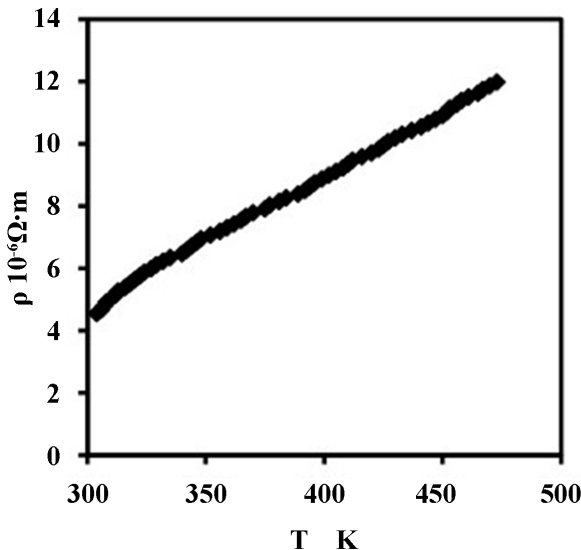

(a)

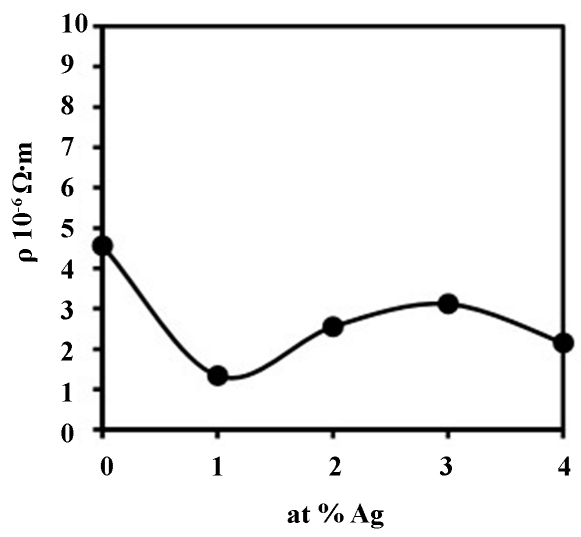

(c)

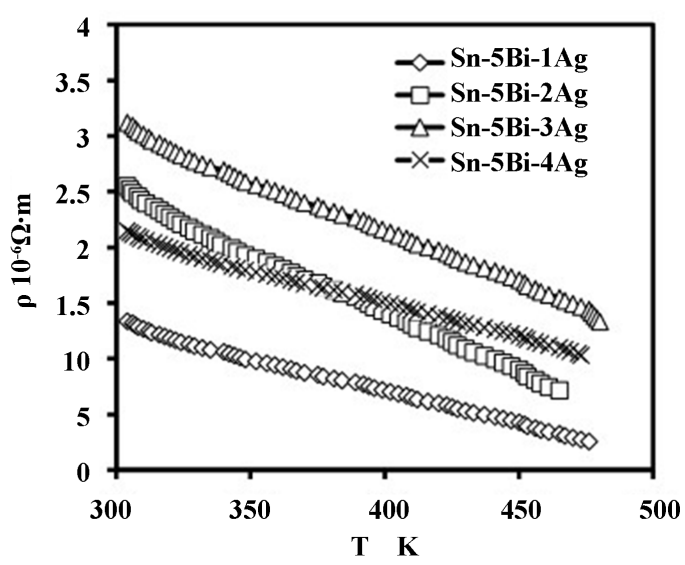

(b)

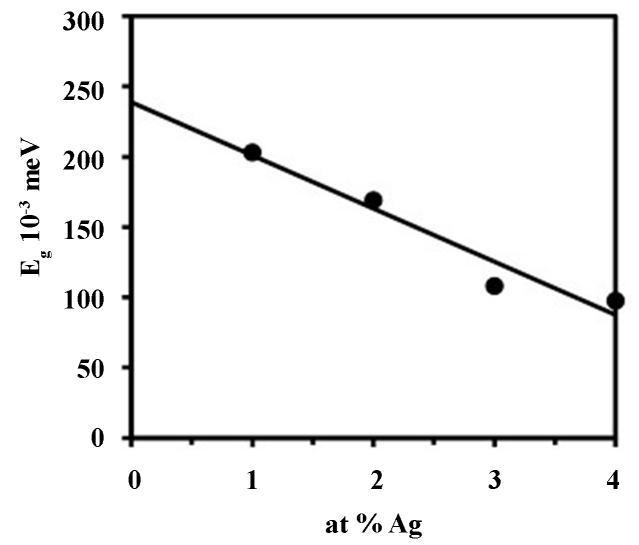

(d)

Figure 4. (a) The TDR for Sn-5Bi alloy; (b) The TDR for as-quenched melt-spun Sn-5Bi-xAg (x = 1, 2, 3, and 4 in at \%) alloys; (c) The variation of $\rho$ at room temperature with Ag concentration; (d) The variation of $\mathrm{E}_{\mathrm{g}}$ with Ag concentration. 
Table 2. The DSC details for as-quenched melt spun pure Sn-5Bi-xAg ( $\mathrm{x}=0,1,2,3$, and 4 in at \%) alloys.

\begin{tabular}{ccc}
\hline Alloy & $\mathrm{T}_{\mathrm{m}} \mathrm{K}$ & $\Delta \mathrm{H}_{\mathrm{m}} \mathrm{kJ}^{\mathrm{kg}} \mathrm{kg}^{-1}$ \\
Sn-5Bi & 492.29 & 57.27 \\
Sn-5Bi-1Ag & 488.88 & 48.58 \\
Sn-5Bi-2Ag & 485.64 & 52.92 \\
Sn-5Bi-3Ag & 485.70 & 47.50 \\
Sn-5Bi-4Ag & 483.58 & 61.09 \\
\hline
\end{tabular}

Table 3. The results of electrical measurements for as-quenched melt-spun Sn-5Bi-x $\operatorname{Ag}(\mathrm{x}=0,1,2,3$, and 4 in at \%) alloys.

\begin{tabular}{ccccc}
\hline Alloy & $\rho \times 10^{-6} \Omega \mathrm{m}$ at room temp. & Behavior & $\mathrm{TCR} \times 10^{-3}\left(\mathrm{~K}^{-1}\right)$ & $\mathrm{E}(\mathrm{meV})$ \\
\hline Sn-5Bi & 4.56 & Metallic & 9.15 & 203 \\
Sn-5Bi-1Ag & 1.34 & Semiconducting & 169 \\
Sn-5Bi-2Ag & 2.55 & Semiconducting & 108 \\
Sn-5Bi-3Ag & 3.12 & Semiconducting & 97.5 \\
Sn-5Bi-4Ag & 2.15 & Semiconducting & & \\
\hline
\end{tabular}

\section{Conclusion}

From above, it is clear that rapidly solidified semiconductors based on Sn-Bi can be produced after the ternary addition of Ag. Since from temperature dependence of electrical resistivity (TDR), it is found that the Sn-5Bi$\mathrm{xAg}(\mathrm{x}=1,2,3,4$ in at \%) rapidly solidified by melt spinning technique are narrow band semiconductor alloys. The energy gap $\mathrm{E}_{\mathrm{g}}$ decreases by increasing $\mathrm{Ag}$ concentration from $203 \mathrm{meV}$ for $\mathrm{Sn}-5 \mathrm{Bi}-1 \mathrm{Ag}$ to $97.5 \mathrm{meV}$ for Sn-5Bi-4Ag. Also from X-ray diffraction analysis (XRD), it is found that Hume-Rothery condition for phase stability is not satisfied for this alloy.

\section{References}

[1] Horio, Y., Yamashita, H. and Hayashi, T. (2004) Microstructure and Thermoelectric Properties of Hot-Pressed p-Type $\mathrm{Bi}_{0.5} \mathrm{Sb}_{1.5} \mathrm{Te}_{3}$ Alloys Prepared by Rapid Solidification Technique. Materials Transactions, 45, 3309-3313. http://dx.doi.org/10.2320/matertrans.45.3309

[2] Raouf A., Kamal M., El Ashram T. and Mosaad S. (2010) Rapidly Solidified Semiconducting Bi-Ag Alloys Produced Using Melt-Spinning Technique. Journal of Ovonic Research, 6, 297-302.

[3] El-Ashram T., Kamal, M., Raouf, M.A. and Mosaad, S. (2012) Odd Valency Dopants Convert Bismuth into Semiconductor. Journal of Ovonic Research, 8, 97-104.

[4] EL-Ashram T. (2015) Formation of Supersaturated Solid Solutions Bi-Ag and Bi-Zn by Rapid Solidification Using Melt Spinning Technique. Materials Sciences and Applications, 6, 183-188. http://dx.doi.org/10.4236/msa.2015.62021

[5] Dutta, S., Shubha, V., Ramesh, T.G. and D’Sa, F. (2009) Thermal and Electronic Properties of Bi1-xSbx Alloys. Journal of Alloys and Compounds, 467, 305-309. http://dx.doi.org/10.1016/j.jallcom.2007.11.146

[6] Lenoira, B., Dauscher, A., Cassartb, M., Ravichc, Y.I. and Scherrer, H. (1998) Effect of Antimony Content on the Thermoelectric Figure of Merit of Bi1-xSbx Alloys. Journal of Physics and Chemistry of Solids, 59, 129-134. http://dx.doi.org/10.1016/S0022-3697(97)00187-X

[7] Lenoira, B., Cassartb, M., Michenaud, J.P., Scherrer, H. and Scherrer, S. (1996) Transport Properties of Bi-rich Bi-Sb Alloys. Journal of Physics and Chemistry of Solids, 57, 89-99. http://dx.doi.org/10.1016/0022-3697(95)00148-4

[8] Kamal, M. and El-Ashram, T. (2008) Zero and Negative Temperature Coefficients of Resistivity of Rapidly Solidified Bi-Sn Alloys Using Melt-Spinning Technique. Journal of Materials Science: Materials in Electronics, 19, 91-96. http://dx.doi.org/10.1007/s10854-007-9315-4

[9] El-Ashram T. (2006) The Structure and Properties of Rapidly Solidified Pure Tin. Radiation Effects and Defects in Solids, 161, 193-197.

[10] El-Ashram T. (2005) The Relation between Valency, Axial Ratio, Young’s Modulus and Resistivity of Rapidly Solidified Tin-Based Eutectic Alloys. Journal of Materials Science: Materials in Electronics, 16, 501-505. http://dx.doi.org/10.1007/s10854-005-2724-3 